\title{
Carbapenem-resistant Acinetobacter baumannii contamination in an intensive care unit
}

\author{
Otávio Hallal Ferreira Raro[1], Stephanie Wagner Gallo[1], \\ Carlos Alexandre Sanchez Ferreira ${ }^{[1]}$ and Sílvia Dias de Oliveira ${ }^{[1]}$
}

[1]. Laboratório de Imunologia e Microbiologia, Faculdade de Biociências, Pontifícia Universidade Católica do Rio Grande do Sul, Porto Alegre, RS, Brasil.

\begin{abstract}
Introduction: Acinetobacter baumannii is a major pathogen causing infections in intensive care units (ICUs). In this study, we aimed to evaluate the presence of $A$. baumannii in an ICU environment and gloves from ICU workers and to characterize the antimicrobial resistance of the isolates in comparison with those isolated from ICU patients at the same hospital. Methods: ICU samples were collected from March to November 2010. Isolates biochemically characterized as Acinetobacter calcoaceticusAcinetobacter baumannii complex were evaluated by PCR targeting the 16S rDNA and bla ${ }_{\mathrm{OXA}-51}$ genes. Antimicrobial susceptibility was determined using the disk diffusion method, and carbapenem-resistant isolates were also evaluated for the minimum inhibitory concentration of imipenem using broth microdilution. The presence of the bla $a_{\mathrm{OX}-23}$ gene was evaluated in isolates with reduced susceptibility to carbapenems. Results: $A$. baumannii was detected in $9.5 \%$ (84) of the 886 samples collected from the ICU environment, including from furniture, medical devices, and gloves, with bed rails being the most contaminated location $(23.8 \%$; 20/84). Multidrug-resistant (MDR) A. baumannii was found in $98.8 \%(83 / 84)$ of non-clinical and $97.8 \%(45 / 46)$ of clinical isolates. Reduced susceptibility to carbapenems was detected in $83.3 \%(70 / 84)$ of non-clinical and $80.4 \%$ (37/46) of clinical isolates. All isolates resistant to carbapenems harbored bla ${ }_{\text {OXA-23 }}$. Conclusions: We found a strong similarity between the antimicrobial susceptibility profiles of non-clinical and clinical $A$. baumannii isolates. Such data highlight the ICU environment as a potential origin for the persistence of MDR A. baumannii, and hence the ICU may be a source of hospital-acquired infections caused by this microorganism.
\end{abstract}

Keywords: Acinetobacter baumannii. Antimicrobial resistance. Carbapenems.

Hospital environmental contamination. Hospital infections.

\section{INTRODUCTION}

Acinetobacter baumannii is an important opportunistic pathogen commonly associated with several health careassociated infections that affect debilitated patients admitted to intensive care units (ICUs) ${ }^{1,2}$. Besides being able to survive in dry environments for a long time, this microorganism can spread by air, water droplets over short distances, the peeling skin of colonized patients ${ }^{3}$, and the hands of hospital workers. Hands carrying pathogens are the main source of the spread of infectious agents, even though hand hygiene is a simple, standardized, and low-cost measure ${ }^{4-6}$. Furthermore, infections caused by A. baumannii in ICUs may be related to the lack of environmental surface cleanliness and the continuous use of medical devices, such as endotracheal tubes and intravascular and urinary catheters, which lead patients to be more susceptible to intensive handling by healthcare workers ${ }^{7}$.

Corresponding author: Dra. Sílvia Dias de Oliveira.

e-mail: silviadias@pucrs.br

Received 22 August 2016

Accepted 8 February 2017
The persistence of $A$. baumannii in a hospital environment exposes it to constant selective pressure imposed by antibiotics, which, in addition to its remarkable ability to acquire resistance determinants, has led to the emergence of multidrug-resistant (MDR) A. baumannii ${ }^{8}$. Resistance to carbapenems has been reported worldwide ${ }^{9-11}$, causing special concern because it is the chosen drug to treat infections caused by A. baumannii. Carbapenem resistance in A. baumannii is mainly mediated by the production of class $\mathrm{D} \beta$-lactamases (oxacillinases) ${ }^{12}$, particularly via the acquisition of genes encoding OXA-23, OXA-24/40, OXA-58, OXA-143, and OXA-51, which are intrinsically located on the chromosome ${ }^{13,14}$. The $b l a_{\mathrm{OXA}-23}$ gene was originally identified on the chromosome of Acinetobacter radioresistens ${ }^{15}$ and is currently widespread in $A$. baumannii, where it is associated with different genetic structures in the chromosome and plasmids ${ }^{10,16,17}$. In this context, this study evaluated the presence of $A$. baumannii in an ICU environment and gloves from ICU workers, characterized the antimicrobial resistance of the isolates obtained from different ICU locations, and compared these with clinical isolates from patients in the same ICU. 


\section{METHODS}

\section{Samples}

Samples were collected weekly during the period from March to November 2010 in an adult ICU of a 603-bed university hospital in Porto Alegre, Southern Brazil, resulting in 886 samples from the hospital environment $(\mathrm{n}=858)$ and from the gloves $(n=28)$ of healthcare workers. Floor samples $(n=33)$ from hospital rooms were collected using a sterile drag swab (4" $\times 4$ " gauze pads) moistened with $0.1 \%$ saline peptone, which was rolled over an area of approximately $8 \mathrm{~m}^{2}$. The gloves were collected and transported in sterilized plastic bags, and remaining samples were collected with a sterile swab moistened with $0.1 \%$ saline peptone, which was rolled once over the entire exposed surface to be sampled. All samples were collected by the same individual. The Department of Microbiology of the Clinical Pathology Laboratory of the hospital provided 46 strains of Acinetobacter calcoaceticus-Acinetobacter baumannii complex from the following clinical specimens: biliary secretion $(\mathrm{n}=$ $1)$, bronchial lavage $(n=5)$, bronchoalveolar lavage $(n=2)$, central venous access $(n=4)$, nasopharyngeal aspirate $(n=2)$, oropharyngeal secretion $(n=3)$, peripheral venous access $(n=4)$, pleural liquid $(n=1)$, skin biopsy $(n=1)$, $\operatorname{sputum}(n=12)$, tracheal aspirate $(n=8)$, tracheal secretion $(n=1)$, and urine $(n=2)$. The clinical specimens included in the study were isolated during the same period in which the environmental samples were collected.

\section{Isolation and identification}

Swabs collected from the hospital environment were placed in $3 \mathrm{~mL}$ BHI broth (Himedia, India) and incubated at $37^{\circ} \mathrm{C}$ with constant rotation (150rpm) for $24 \mathrm{~h}$ according to Baumann ${ }^{18}$ and were then spread on MacConkey agar (Oxoid, England). An aliquot of $5 \mathrm{~mL}$ nutrient broth was added to the inside of each glove, homogenized, and incubated for $5 \mathrm{~min}$ at $22-25^{\circ} \mathrm{C}$. After this, $100 \mu \mathrm{L}$ was spread on blood agar (Himedia, India) and incubated at $37^{\circ} \mathrm{C}$ for $24 \mathrm{~h}$. A volume of $100 \mu \mathrm{L}$ saline peptone obtained from each floor sample was spread on blood agar and incubated at $37^{\circ} \mathrm{C}$ for $24 \mathrm{~h}$. Colonies were preliminarily screened by morphology and characterized by oxidase (Laborclin, Brazil) and triple sugar iron (Oxoid, England) tests. All tests were standardized using the reference culture A. baumannii ATCC 19606.

Isolates presenting a biochemical profile compatible with A. calcoaceticus-baumannii complex were subjected to deoxyribonucleic acid (DNA) extraction using guanidine isothiocyanate according to Rademaker and de Brujin ${ }^{19}$. Purified DNA was used as a template for polymerase chain reaction (PCR) targeting the $16 \mathrm{~S}$ ribosomal deoxyribonucleic acid $(\mathrm{rDNA})^{20}$ and bla $_{\mathrm{OXA}-51}$ genes to identify Acinetobacter spp. and $A$. baumannii, respectively. Primers targeting $b l a_{\text {OXA-51 }}$ were designed in this study (5'-GACGGGCAAAAAAGGCTA-3' and 5'-GGGTCTACATCCCATCCC-3') (Integrated DNA Technologies, USA) to amplify a $347-b p$ fragment. PCR amplifications were performed in a final volume of $25 \mu \mathrm{L}$ containing $1 \mu \mathrm{L}$ target DNA, $0.2 \mathrm{mM}$ each deoxynucleoside triphosphate, $50 \mathrm{mM} \mathrm{KCl}, 10 \mathrm{mM}$ Tris- $\mathrm{HCl}$ (pH 8.3), $1.5 \mathrm{mM}$ $\mathrm{MgCl}_{2}, 1 \mathrm{U}$ Taq DNA polymerase (Invitrogen Life Technologies, USA), and $0.8 \mu \mathrm{M}$ each primer. Amplifications were carried out in a thermocycler (MiniCycler ${ }^{\mathrm{TM}}$, MJ Research, USA) using the following conditions: initial denaturation at $95^{\circ} \mathrm{C}$ for $5 \mathrm{~min}$; followed by 35 cycles of denaturation at $95^{\circ} \mathrm{C}$ for $1 \mathrm{~min}$, annealing at $51^{\circ} \mathrm{C}$ for $1 \mathrm{~min}$, and extension at $72^{\circ} \mathrm{C}$ for $1 \mathrm{~min}$ and $30 \mathrm{~s}$; with a final extension at $72^{\circ} \mathrm{C}$ for $10 \mathrm{~min}$. Positive and negative controls were included using DNA from $A$. baumannii ATCC 19606 and a DNA-free reaction, respectively. Amplicons were analyzed by electrophoresis using agarose gels stained with $0.5 \mu \mathrm{g} / \mathrm{mL}$ ethidium bromide and visualized under $\mathrm{UV}$ radiation. The amplification products targeting $b l a_{\text {OXA-51 }}$ from two isolates were purified with ammonium acetate and sequenced in an automated DNA sequencer (ABI $3130 \mathrm{XL}$ Genetic Analyzer, Applied Biosystems, USA). Searches and alignments of nucleotide sequences were performed with the BLAST algorithm (http://www.ncbi.nlm.nih.gov/BLAST).

\section{Antimicrobial susceptibility testing}

The antimicrobial susceptibility of each isolate was determined using the disk diffusion method according to the Clinical and Laboratory Standards Institute (CLSI) guidelines ${ }^{21}$. The antimicrobials tested were the following: amikacin (AMI), ampicillin-sulbactam (AMS), aztreonam (ATM), cefepime (CPM), cefotaxime (CTX), ceftazidime (CAZ), ceftriaxone (CRO), ciprofloxacin (CIP), doxycycline (DX), gentamicin (GEN), imipenem (IPM), levofloxacin (LVX), meropenem (MEM), minocycline (MIN), piperacillin-tazobactam (PTZ), trimethoprim-sulfamethoxazole (COT), tetracycline (TET), ticarcillin-clavulanic acid (TCC), and tobramycin (TOB). MDR was defined as the absence of susceptibility to at least one agent in $\geq 3$ antimicrobial categories ${ }^{22}$.

Carbapenem-resistant isolates characterized by the disk diffusion test were also evaluated regarding the minimum inhibitory concentration (MIC) of imipenem by broth microdilution, which was interpreted according to the CLSI guidelines ${ }^{21}$. All antimicrobial susceptibility analyses were performed using Escherichia coli ATCC 25922 and Pseudomonas aeruginosa ATCC 27853 as reference strains for antibiotic quality control.

\section{Detection of bla $_{\text {OXA-23 }}$}

Isolates that showed reduced susceptibility to carbapenems were evaluated for the presence of the bla $a_{\text {OXA-23 }}$ gene by PCR as previously described ${ }^{23}$, with the exception of the $\mathrm{MgCl}_{2}$ concentration, which was changed to $2.5 \mathrm{mM}$. In order to ensure the specificity of PCR, one amplification product targeting $b l a_{\text {OXA-23 }}$ was purified and sequenced as described above.

\section{Statistical analysis}

Antimicrobial susceptibility data were analyzed and compared by $\chi^{2}$ test or Fisher's exact test. In order to analyze differences among sources (hospital non-clinical or clinical) in resistance to all antimicrobials tested, drugs were grouped into four clusters based on resistance rates $(0-25 \%, 25-50 \%, 50-75 \%$, and $75-100 \%$ ) and evaluated by Cochran-Mantel-Haenszel test. The data were analyzed using SAS software version 9.4 (SAS Institute Inc., USA), and the level of significance was set at 0.05 for all tests. 


\section{RESULTS}

Acinetobacter baumannii was detected in 9.5\% (84) of the 886 samples collected from the ICU environment, including furniture, medical devices, and gloves, referred to as non-clinical isolates. Among the A. baumannii-contaminated samples, 4.8\% (4) were isolated from the floor and $2.4 \%$ (2) were isolated from gloves. Other strains (78) were isolated mainly from bed rails $(23.8 \%)$, bedside tables (10.7\%), and ambu bags (8.3\%). However, this microorganism could be found in many other locations in the ICU environment, such as the monitors of mechanical ventilators, valves (of oxygen, air, and vacuum), intravenous pumps, door handles, stethoscopes, biological sewage, doors, ventilators, tables for nursing staff support, bedside chairs, equipment and medical carts, biological waste, pressure gauges, overbed tables, vital sign monitors, staff clipboards (for patient information), and serum support apparatuses.

All 84 non-clinical and 46 clinical isolates biochemically compatible with the $A$. calcoaceticus-baumannii complex were confirmed as Acinetobacter spp. and identified as $A$. baumannii by $16 \mathrm{~S}$ rDNA and $b l a_{\text {OXA-51 }}$ PCR, respectively. Two $b l a_{\text {OXA-51 }}$ amplification products were sequenced and showed at least $98 \%$ identity with other $A$. baumannii OXA-51 sequences deposited in GenBank (accession numbers AJ309734.2, KJ584920.1, and KJ584925.1).

The antimicrobial susceptibility profiles of the $A$. baumannii isolates are summarized in Table $\mathbf{1}$. The reduced susceptibility rates (i.e., the sum of full and intermediate resistance rates) of clinical and non-clinical isolates to most drugs were not significantly different $(\mathrm{p}>0.05)$, with the exceptions of ceftazidime and amikacin. Furthermore, both groups of isolates showed the highest resistance rates to the same five antimicrobials (aztreonam, ceftriaxone, cefotaxime, cefepime, and ticarcillin-clavulanic acid), as well as the lowest resistance rates to doxycycline and minocycline.

The results of the clustering analysis of reduced susceptibility rates for all antimicrobials tested within each isolate source (non-clinical or clinical) are shown in Table 2, and significant differences $(p<0.05)$ were found among all clusters (I, II, III, and IV). The results further indicated strong similarity between the non-clinical and clinical clusters in their susceptibility to most drugs, with the exceptions of tetracycline (in clusters I and II for clinical and non-clinical isolates, respectively) and levofloxacin and ceftazidime (in clusters III and IV for nonclinical and clinical isolates, respectively). Non-clinical isolates were resistant to more antimicrobial categories than clinical isolates $(\mathrm{p}<0.05)$.

In this study, 98.8\% (83) of non-clinical and 97.8\% (45) of clinical isolates were classified as MDR. Only one strain isolated from the floor was susceptible to all drugs tested. Among the non-clinical isolates, 49 different resistance profiles were obtained, whereas 33 were obtained from among clinical isolates. The most common profile found among all isolates presented resistance to at least 14 antimicrobials, with the

TABLE 1

Reduced susceptibility rates of hospital non-clinical and clinical Acinetobacter baumannii isolates.

\begin{tabular}{|c|c|c|c|}
\hline \multirow{2}{*}{$\begin{array}{l}\text { Antimicrobial } \\
\text { drugs }\end{array}$} & \multicolumn{3}{|c|}{ Reduced susceptibility rates ${ }^{*}(\%)$} \\
\hline & Non-clinical isolates $(n=84)$ & Clinical isolates $(n=46)$ & p-value \\
\hline MIN & $7(8.3)$ & $2(4.3)$ & 0.49 \\
\hline TCC & $76(89.4)$ & $43(93.5)$ & 0.53 \\
\hline IPM & $68(80)$ & $37(80.4)$ & 1 \\
\hline LVX & $62(72.9)$ & $39(84.8)$ & 0.19 \\
\hline MEM & $70(82.3)$ & $36(78.3)$ & 0.74 \\
\hline CAZ & $45(52.9)$ & $35(76.1)$ & 0.01 \\
\hline TET & $34(40)$ & $10(21.7)$ & 0.05 \\
\hline DX & $4(4.8)$ & $0(0)$ & 0.16 \\
\hline TOB & $55(64.7)$ & $28(60.9)$ & 0.81 \\
\hline CTX & $79(92.9)$ & $45(97.8)$ & 0.42 \\
\hline CIP & $76(89.4)$ & $41(89.1)$ & 1 \\
\hline AMI & $61(72.6)$ & $23(50)$ & 0.01 \\
\hline $\mathrm{CRO}$ & $82(96.5)$ & $45(97.3)$ & 1 \\
\hline CPM & $77(90.6)$ & $44(95.6)$ & 0.49 \\
\hline PTZ & $73(85.9)$ & $40(86.9)$ & 1 \\
\hline ATM & $81(95.3)$ & $46(100)$ & 0.30 \\
\hline GEN & $61(71.8)$ & $32(69.6)$ & 0.95 \\
\hline AMS & $62(72.9)$ & $29(63)$ & 0.33 \\
\hline COT & $74(87.1)$ & $36(78.3)$ & 0.29 \\
\hline
\end{tabular}

MIN: minocycline; TCC: ticarcillin-clavulanic acid; IPM: imipenem; LVX: levofloxacin; MEM: meropenem; CAZ: ceftazidime; TET: tetracycline; DX: doxycycline; TOB: tobramycin; CTX: cefotaxime; CIP: ciprofloxacin; AMI: amikacin; CRO: ceftriaxone; CPM: cefepime; PTZ: piperacillin-tazobactam; ATM: aztreonam; GEN: gentamicin; AMS: ampicillin-sulbactam; COT: trimethoprim-sulfamethoxazole. *Reduced susceptibility rate is the sum of the full and intermediate resistance rates. 
TABLE 2

Clustering of antimicrobials according to the reduced susceptibility rates of each Acinetobacter baumannii source.

\begin{tabular}{lcc}
\hline Clusters & Non-clinical isolates & Clinical isolates \\
\hline I $(0-25 \%)^{*}$ & MIN; DX & TET; MIN; DX \\
II $(25-50 \%)$ & TET & - \\
III $(50-75 \%)$ & TOB; LVX; GEN; CAZ; AMS; AMI & TOB; GEN; AMS; AMI \\
IV (75-100\%) & TCC; PTZ; MEM; IPM; CTX; CRO; CPM; COT; & CIP; ATM \\
& TCC; PTZ; MEM; LVX; IPM; CTX; CRO; \\
\hline
\end{tabular}

MIN: minocycline; TCC: ticarcillin-clavulanic acid; IPM: imipenem; LVX: levofloxacin; MEM: meropenem; CAZ: ceftazidime; TET: tetracycline; DX: doxycycline; TOB: tobramycin; CTX: cefotaxime; CIP: ciprofloxacin; AMI: amikacin; CRO: ceftriaxone; CPM: cefepime; PTZ: piperacillin-tazobactam; ATM: aztreonam; GEN: gentamicin; AMS: ampicillin-sulbactam; COT: trimethoprim-sulfamethoxazole. *Percentages refer to the reduced susceptibility rates of isolates to the antimicrobials tested.

most (11.8\%) prevalent profile among non-clinical isolates characterized by reduced susceptibility to the following 17 drugs: TCC, IPM, LVX, MEM, CAZ, TET, TOB, CTX, CIP, AMI, CRO, CPM, PTZ, ATM, GEN, AMS, and COT. Among the clinical isolates, there were two other profiles that were more prevalent (10.9\% each). One of them presented reduced susceptibility to the same antimicrobials found in the predominant profile of non-clinical isolates, except for the absence of resistance to tetracycline, while the other presented only two differences (absence of resistance to amikacin and tetracycline).

Reduced susceptibility to carbapenems (imipenem and meropenem) was detected among $83.3 \%$ (70) of non-clinical and $80.4 \%$ (37) of clinical isolates, and no significant differences were found in the rates of reduced susceptibility to imipenem ( $p$ $=1)$ or meropenem $(p=0.74)$ between the two groups of isolates. The MIC of imipenem in carbapenem-resistant isolates ranged from $8 \mu \mathrm{g} / \mathrm{mL}$ to $64 \mu \mathrm{g} / \mathrm{mL}$, and all $A$. baumannii isolates resistant to carbapenems harbored the $b l a_{\mathrm{OXA}-23}$ gene. The specificity of the PCR amplification targeting $b l a_{\mathrm{OXA}-23}$ was confirmed by sequencing one amplicon that showed at least $96 \%$ identity with other bla $_{\text {OXA-23 }}$ sequences deposited in GenBank (GenBank accession numbers JN207493.1, GQ861438.1, and AJ132105.1).

\section{DISCUSSION}

The ability of $A$. baumannii to survive for a long time in the hospital environment and to readily acquire antimicrobial resistance has driven increased concern over this species in the medical community ${ }^{2,5}$. In this study, we evaluated the presence of this microorganism in the ICU environment, including furniture, medical devices, and gloves, as well as characterizing the antimicrobial resistance profiles of these isolates and comparing them with those obtained from clinical sources in the same hospital and over the same period. A. baumannii was detected in a wide variety of samples collected from the ICU rooms, indicating the spread of this microorganism across the hospital locations evaluated. The bed rail was the most commonly contaminated location, suggesting it may act as a possible environmental reservoir of $A$. baumannii in the ICU. This reinforces the importance of disinfecting and cleaning protocols for hospital medical devices and surfaces to control and reduce the cross-transmission of $A$. baumannii.
In addition to the ICU environment and medical devices, the hands of healthcare workers are an important mode of dissemination for many pathogens $\mathbf{s}^{24,25}$, acting as a source of cross-infection and re-infection. Thus, we analyzed the insides of gloves used by ICU workers as an indicator of hand contamination, and we found that $7.4 \%$ were contaminated with A. baumannii. Hands may be even more contaminated than this estimate, since the analysis of gloves may underestimate the real prevalence of bacteria on the hands. It is also important to note that as only the inside of each glove was sampled, we cannot evaluate the possible transmission of A. baumannii via gloves, but this may certainly be occurring, especially considering the presence of $A$. baumannii in various locations in the ICU.

The surfaces of the hospital environment have been proposed to act as reservoirs of MDR bacteria for patient-to-patient transmission ${ }^{26}$. In this context, we found a strong similarity in the antimicrobial susceptibility profiles of non-clinical and clinical A. baumannii isolates, reinforcing the idea that many locations in ICU rooms must be observed with considerable concern. Both clinical and non-clinical isolates showed high rates of antimicrobial multidrug resistance, especially when compared to other studies of clinical isolates that used the same criteria to define $\mathrm{MDR}^{27-29}$.

The low susceptibility rates to carbapenems detected among both clinical and non-clinical isolates from the ICU environment indicates a significant limitation in the treatment options for Acinetobacter infections, particularly when resistance is mediated by mobilizable determinants that can be promptly transferred and spread between isolates ${ }^{30,31}$. All carbapenem-resistant $A$. baumannii isolated in this study harbored the $b l a_{\mathrm{OXA}-23}$ gene, as has been also reported by other authors worldwide ${ }^{10,32-35}$. The high prevalence of $b l a_{\text {OXA-23 }}$ may be due to its horizontal transfer, since it has been also identified on plasmids ${ }^{16,36}$. Moreover, the presence of $b l a_{\text {OXA-23 }}$ may constitute a larger problem, as in addition to its ability to hydrolyze carbapenems, OXA-23 is involved in the resistance to ticarcillin and amoxicillin, which not only affects patient care but possibly also increases resistance selection rates ${ }^{31}$. In contrast, minocycline and doxycycline are therapeutic options for the treatment of carbapenem-resistant $A$. baumannii infections. Minocycline and doxycycline have already been 
found to be effective against Acinetobacter spp., with isolates presenting susceptibility rates of $80-90 \%{ }^{37,38}$. In this sense, minocycline has been used successfully for the treatment of wound infections and ventilator-associated pneumonia caused by MDR $A$. baumannii $i^{39}$.

The high resistance rates found among non-clinical isolates are of great concern since they identify the ICU environment as a possible major reservoir of resistant $A$. baumannii and, consequently, a source of healthcare-associated infections. Thus, it should be emphasized that hospital infection prevention and control measures, such as intensified hand hygiene adherence, patient cohorts or isolation, active surveillance, and especially better cleaning and disinfection of the environment may prevent outbreaks of MDR bacteria in ICUs.

\section{Acknowledgements}

We thank João Riboldi by statistical analysis support, the Clinical Pathology Laboratory of the Hospital São Lucas for sending A. baumannii from clinical specimens, and the Infection Control Service and workers of the ICU for their help during the ICU sampling.

\section{Conflicts of interest}

The authors declare that there is no conflict of interest.

\section{Financial support}

The first author received a scholarship from the Coordination for the Improvement of Higher Education Personnel (Coordenação de Aperfeiçoamento de Pessoal de Nível Superior - CAPES), Brazil.

\section{REFERENCES}

1. Centers for Disease Control and Prevention (CDC). Acinetobacter in Healthcare Settings. 2014. Accessed 14 March 2016. Available from: http://www.cdc.gov/HAI/organisms/organisms.html.

2. Peleg AY, Seifert H, Paterson DL. Acinetobacter baumannii: emergence of a successful pathogen. Clin Microbiol Rev. 2008;21(3):538-82.

3. Bernards AT, Frénay HM, Lim BT, Hendriks WD, Dijkshoorn L, van Boven CP. Methicillin-resistant Staphylococcus aureus and Acinetobacter baumannii: an unexpected difference in epidemiologic behavior. Am J Infect Control. 1998;26(6): 544-51.

4. Chen YC, Sheng WH, Wang JT, Chang SC, Lin HC, Tien KL, et al. Effectiveness and limitations of hand hygiene promotion on decreasing healthcare-associated infections. PLoS ONE. 2011;6(11):e27163. doi: 10.1371/journal.pone.0027163.

5. Dijkshoorn L, Nemec A, Seifert H. An increasing threat in hospitals: multidrug-resistant Acinetobacter baumannii. Nat Rev Microbiol. 2007;5(12):939-51.

6. Morgan DJ, Liang SY, Smith CL, Johnson JK, Harris AD, Furuno JP, et al. Frequent multidrug-resistant Acinetobacter baumannii contamination of gloves, gowns, and hands of healthcare workers. Infect Control Hosp Epidemiol. 2010;31(7):716-21.

7. Alfandari S, Gois J, Delannoy PY, Georges H, Boussekey N, Chiche A, et al. Management and control of a carbapenem-resistant Acinetobacter baumannii outbreak in an intensive care unit. Med Mal Infect. 2014;44(5):229-31.

8. Lob SH, Hoban DJ, Sahm DF, Badal RE. Regional differences and trends in antimicrobial susceptibility of Acinetobacter baumannii. Int J Antimicrob Agents. 2016;47(4):317-23.
9. Higgins PG, Dammhayn C, Hackel M, Seifert H. Global spread of carbapenem-resistant Acinetobacter baumannii. J Antimicrob Chemother. 2010;65(2):233-8.

10. Vasconcelos AT, Barth AL, Zavascki AP, Gales AC, Levin AS, Lucarevschi BR, et al. The changing epidemiology of Acinetobacter spp. producing OXA carbapenemases causing bloodstream infections in Brazil: a BrasNet report. Diagn Microbiol Infect Dis. 2015;83(4):382-5.

11. Zilberberg MD, Kollef MH, Shorr AF. Secular trends in Acinetobacter baumannii resistance in respiratory and blood stream specimens in the United States, 2003 to 2012: A survey study. J Hosp Med. 2016;11(1):21-6.

12. Poirel L, Naas T, Nordmann P. Diversity, epidemiology, and genetics of class D beta-lactamases. Antimicrob Agents Chemother. 2010;54(1):24-38.

13. Mendes RE, Bell JM, Turnidge JD, Castanheira M, Jones RN. Emergence and widespread dissemination of OXA-23, -24/40 and -58 carbapenemases among Acinetobacter spp. in AsiaPacific nations: report from the SENTRY Surveillance Program. J Antimicrob Chemother. 2009;63:55-9.

14. Zander E, Fernández-González A, Schleicher X, Dammhayn C, Kamolvit W, Seifert H, et al. Worldwide dissemination of acquired carbapenem-hydrolysing class D $\beta$-lactamases in Acinetobacter spp. other than Acinetobacter baumannii. Int J Antimicrob Agents. 2014;43(4):375-7.

15. Poirel L, Figueiredo S, Cattoir V, Carattoli A, Nordmann P. Acinetobacter radioresistens as a silent source of carbapenem resistance for Acinetobacter spp. Antimicrob Agents Chemother. 2008;52(4):1252-6.

16. Mugnier PD, Poirel L, Naas T, Nordmann P. Worldwide dissemination of the bla ${ }_{\mathrm{OXA}-23}$ carbapenemase gene of Acinetobacter baumannii. Emerg Infect Dis. 2010;16(1):35-40.

17. Pagano M, Barin J, Martins AF, Zavascki AP. High endemic rates of OXA-23-producing carbapenem-resistant Acinetobacter baumannii isolates caused by the persistence of major clones in hospitals in a Brazilian city 5 years after an outbreak. Infect Control Hosp Epidemiol. 2015;36(7):860-2.

18. Baumann P. Isolation of Acinetobacter from soil and water. J Bacteriol. 1968;96(1):39-42.

19. Rademaker JLW, de Bruijin FJ. Characterization and classification of microbes by REP-PCR genomic fingerprinting and computerassisted pattern analysis. In: Caetano-Anollés G, Gresshoff PM, editors. DNA markers: protocols, applications and overviews. New York: John Wiley and Sons; 1997. p. 151-71.

20. Turton JF, Kaufmann ME, Glover J, Coelho JM, Warner M, Pike $\mathrm{R}$, et al. Detection and typing of integrons in epidemic strains of Acinetobacter baumannii found in the United Kingdom. J Clin Microbiol. 2005; 43(7):3074-82.

21. Clinical and Laboratory Standards Institute (CLSI). Performance Standards for Antimicrobial Susceptibility Testing; TwentyFifth Informational Supplement (M100-S25). CLSI Document. Pennsylvania: Wayne; 2015.

22. Magiorakos AP, Srinivasan A, Carey RB, Carmeli Y, Falagas ME, Giske CG, et al. Multidrug-resistant, extensively drug-resistant and pandrug-resistant bacteria: an international expert proposal for interim standard definitions for acquired resistance. Clin Microbiol Infect. 2012;18(3):268-81.

23. Donald HM, Scaife W, Amyes SG, Young HK. Sequence analysis of ARI-1, a novel OXA beta-lactamase, responsible for imipenem resistance in Acinetobacter baumannii 6B92. Antimicrob Agents Chemother. 2000;44(1):196-9. 
24. Dancer SJ. Importance of the environment in meticillin-resistant Staphylococcus aureus acquisition: the case for hospital cleaning. Lancet Infect Dis. 2008;8(2):101-13.

25. Sax H, Allegranzi B, Uçkay I, Larson E, Boyce J, Pittet D. "My five moments for hand hygiene": a user-centred design approach to understand, train, monitor and report hand hygiene. J Hosp Infect. 2007;67(1):9-21.

26. Hess AS, Shardell M, Johnson JK, Thom KA, Roghmann MC, Netzer G, et al. A randomized controlled trial of enhanced cleaning to reduce contamination of healthcare worker gowns and gloves with multidrug-resistant bacteria. Infect Control Hosp Epidemiol. 2013;34(5):487-93.

27. McCracken M, Mataseje LF, Loo V, Walkty A, Adam HJ, Hoban DJ, et al. Characterization of Acinetobacter baumannii and meropenem-resistant Pseudomonas aeruginosa in Canada: results of the CANWARD 2007-2009 study. Diagn Microbiol Infect Dis. 2011;69(3):335-41.

28. Rivera G, Bulnes J, Castillo C, Ajenjo MC, Garcia P, Labarca J. Extensively drug-resistant Acinetobacter baumannii isolated in a university hospital: role of inter-hospital transmission. J Infect Dev Ctries. 2016;10(1):96-9.

29. Wadl M, Heckenbach K, Noll I, Ziesing S, Pfister W, Beer J, et al. Increasing occurrence of multidrug-resistance in Acinetobacter baumannii isolates from four German University Hospitals, 20022006. Infection. 2010;38(1) 47-51.

30. Perez F, Hujer AM, Hujer KM, Decker BK, Rather PN, Bonomo RA. Global challenge of multidrug-resistant Acinetobacter baumannii. Antimicrob Agents Chemother. 2007;51(10):3471-84.

31. Evans BA, Hamouda A, Amyes SG. The rise of carbapenem-resistant Acinetobacter baumannii. Curr Pharm Des. 2013;19(2):223-38.
32. Ferreira AE, Marchetti DP, De Oliveira LM, Gusatti CS, Fuentefria DB, Corção G. Presence of OXA-23-producing isolates of Acinetobacter baumannii in wastewater from hospitals in Southern Brazil. Microb Drug Resist. 2011;17(2):221-7.

33. Niumsup PR, Boonkerd N, Tansawai U, Tiloklurs M. Carbapenemresistant Acinetobacter baumannii producing OXA-23 in Thailand. Jpn J Infect Dis. 2009;62(2):152-4.

34. Stoeva T, Higgins PG, Savov E, Markovska R, Mitov I, Seifert H. Nosocomial spread of OXA-23 and OXA-58 beta-lactamaseproducing Acinetobacter baumannii in a Bulgarian hospital. J Antimicrob Chemother. 2009;63(3):618-20.

35. Zhou H, Pi BR, Yang Q, Yu YS, Chen YG, Li LJ, et al. Dissemination of imipenem-resistant Acinetobacter baumannii strains carrying the ISAbal-bla $a_{\mathrm{OXA}-23}$ genes in a Chinese hospital. J Med Microbiol. 2007;56(8):1076-80.

36. Scaife W, Young HK, Paton RH, Amyes SG. Transferable imipenem-resistance in Acinetobacter species from a clinical source. J Antimicrob Chemother. 1995;36(3):585-6.

37. Akers KS, Mende K, Yun HC, Hospenthal DR, Beckius ML, Yu $\mathrm{X}$, et al. Tetracycline susceptibility testing and resistance genes in isolates of Acinetobacter baumannii-Acinetobacter calcoaceticus complex from a U.S. military hospital. Antimicrob Agents Chemother. 2009;53(6):2693-5.

38. Valentine SC, Contreras D, Tan S, Real LJ, Chu S, Xu HH. Phenotypic and molecular characterization of Acinetobacter baumannii clinical isolates from nosocomial outbreaks in Los Angeles County, California. J Clin Microbiol. 2008;46(8):2499-507.

39. Griffith ME, Yun HC, Horvath LL, Murray CK. Minocycline therapy for traumatic wound infections caused by the multidrugresistant Acinetobacter baumannii-Acinetobacter calcoaceticus complex. Infect Dis Clin Pract. 2008;16(1):16-19. 